\title{
On the Effects of Channel Aging in D2D Two-Way Relaying with Space-Constrained Massive MIMO
}

\author{
Jun Qian, Christos Masouros \\ The Department of Electronic and Electrical Engineering \\ University College London \\ Gower Street, London, WC1E 6BT \\ Email: \{jun.qian.15, c.masouros\}@ucl.ac.uk
}

\begin{abstract}
This paper studies the spectral efficiency (SE) of a space-constrained multi-pair two-way massive multiple-input multiple-output (MIMO) decode-and-forward (DF) relay system with channel aging for device-to-device (D2D) communications in the Internet of Things (IoT) environments. Maximum ratio combining/Maximum ratio transmission (MRC/MRT) processing is employed at the relay and imperfect channel estimation is assumed. With the consideration of the spatial correlation due to insufficiently spaced antennas, and the time correlation due to channel aging, we study the closed-form large-scale approximations of the SE performance. Our analytical studies and performance results demonstrate that a degree of both spatial correlation due to antenna proximity, and time correlation due to channel aging can be tolerated in the massive MIMO regime without significant performance degradation.

Index Terms-Channel aging, Decode-and-forward relaying, D2D communications, maximum ratio combining/transmission, spatial correlation, spectral efficiency.
\end{abstract}

\section{INTRODUCTION}

With the rise of IoT applications, device-to-device (D2D) communications have attracted great interest. The deployment of a two-way relay provides an attractive solution to facilitate D2D communications between multiple resource limited device pairs [1], [2]. Multi-pair D2D two-way relaying allows device pairs to exchange information with each other simultaneously with the aid of a shared relay and has been proven as a useful method to improve the spectral efficiency (SE) performance [3], [4]. Generally, the amplify-and-forward (AF) protocol has been widely addressed in previous studies of multi-pair two-way relaying. However, the AF relaying could suffer from noise amplification. Accordingly, the decode-andforward (DF) relaying which could achieve better performance than AF relaying in scenarios with low signal-to-noise ratios (SNRs), is considered in this paper [2], [5], [6].

Moreover, the proven SE benefits of massive multiple-input multiple-output (MIMO) have promoted its use in the multipair two-way relay systems [7], [8]. Multi-pair two-way massive MIMO relaying with imperfect channel state information (CSI) has been studied since perfect CSI is impossible in most practical scenarios [6], [9]. Additionally, channel aging making the estimated CSI out of date has been introduced in the multipair massive MIMO relay system, most of the relevant studies

This work was supported by the Engineering and Physical Sciences Research Council (EPSRC) project EP/M014150/1. are operated in full-duplex AF mode [3], [10], [11] or halfduplex AF mode [10], while multi-pair D2D two-way halfduplex DF relay system with channel aging is overlooked.

Motivated by the above, we investigate the SE performance of a multi-pair D2D two-way massive MIMO half-duplex DF relaying system with imperfect CSI and channel aging. In addition, employing large antenna arrays in constrained space leads to the consideration of spatial correlation [12], [13], which is rarely considered in the multi-pair half-duplex DF two-way relayed D2D communication systems. To this end, based on the studies in [9], a detailed analysis of the achievable SE performance of a multi-pair D2D two-way massive MIMO half-duplex relay system under DF protocol considering both the spatial correlation due to the space-constrained antenna deployment and the time correlation due to channel aging is presented.

\section{SYSTEM MODEL}

\section{A. Channel Model}

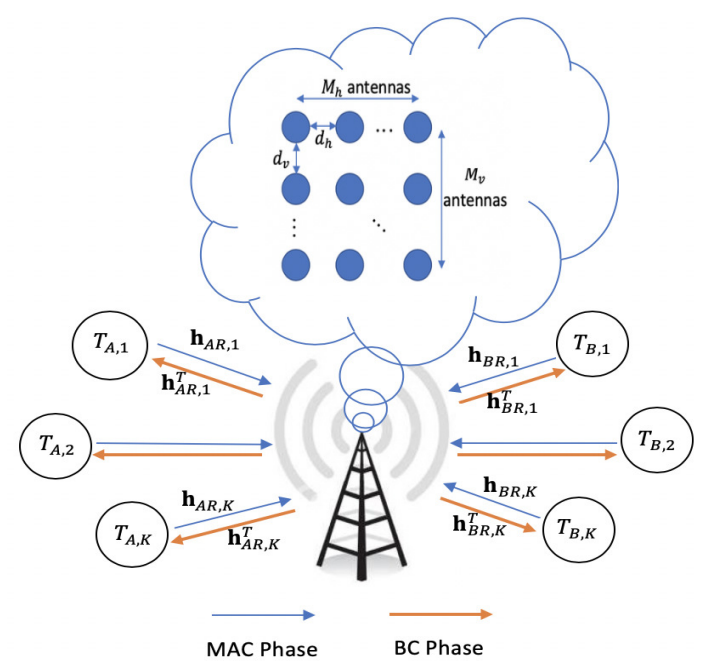

Fig. 1. Multi-pair D2D two-way half-duplex DF relaying system with spaceconstrained massive MIMO.

As shown in Fig. 1, we assume that $K$ single-antenna device pairs, denoted as $\mathrm{T}_{A, k}$ and $\mathrm{T}_{B, k}, k=1, \ldots, K$, communicate with each other via the shared relay $\mathrm{T}_{R}$ with $M$ closely-spaced 


$$
\mathbf{a}\left(\theta_{k, i}, \phi_{k, i}\right)=\left[1, e^{j 2 \pi\left[d_{h} \sin \left(\phi_{k, i}\right) \sin \left(\theta_{k, i}\right)\right]}, \ldots, e^{j 2 \pi\left[\left(M_{h}-1\right) d_{h} \sin \left(\phi_{k, i}\right) \sin \left(\theta_{k, i}\right)+\left(M_{v}-1\right) d_{v} \sin \left(\phi_{k, i}\right) \cos \left(\theta_{k, i}\right)\right]}\right]
$$

antennas $(M \gg K)$ with less than half-wavelength distance and there is no direct link between device pairs. Following the closely related literature [14], [15], the proposed system is modeled as spatially-correlated Rayleigh fading under TDD mode with channel reciprocity. To this end, $\mathbf{h}_{X R, k}$ and $\mathbf{h}_{X R, k}^{T}$ are defined as the uplink and downlink channels between $\mathrm{T}_{X, k},(X=A, B, k=1, \ldots, K)$ and $\mathrm{T}_{R}$, respectively. The uplink channel can be given by [12], [16]

$$
\mathbf{h}_{X R, k}=\mathbf{A}_{k}^{H} \mathbf{g}_{X R, k},
$$

where $\mathbf{g}_{X R, k} \sim \mathcal{C N}\left(0, \beta_{X R, k} \mathbf{I}_{L_{k}}\right), L_{k}$ is the number of angular directions and we consider $L_{k}=L(k=1, \ldots, K)$ in the simulations for simplicity. $\beta_{A R, k}$ and $\beta_{B R, k}$ represent the large-scale fading effects. $\mathbf{A}_{k} \in \mathbb{C}^{L \times M}$, the transmit steering matrix at $T_{X, k}$, can be expressed as [16], [17]

$$
\mathbf{A}_{k}=\frac{1}{\sqrt{L}}\left[\mathbf{a}^{T}\left(\theta_{k, 1}, \phi_{k, 1}\right), \ldots, \mathbf{a}^{T}\left(\theta_{k, L}, \phi_{k, L}\right)\right]^{T},
$$

here, $\theta_{k, i}$ and $\phi_{k, i}, i=1, \ldots, L$ denote respective azimuth and elevation angles of departure. The steering vector $\mathbf{a}\left(\theta_{k, i}, \phi_{k, i}\right)$ for the uniform rectangular array (URA) topology can be given on the top of this page [12], where $M_{h}$ and $M_{v}$ stand for the horizontal and vertical number of antennas respectively with $M=M_{h} \times M_{v}$. Meanwhile, $d_{h}$ and $d_{v}$ represent the horizontal and vertical inter-antenna distance normalized by the carrier wavelength $\lambda$, respectively. For the sake of simplicity, we consider that $d_{h}=d_{v}=d$ in the following. It is also readily assumed that there is no receive correlation since the interdevice distance is greater than $\lambda[12]$.

\section{B. Channel Aging}

Similar to [3], [10], [11], we assume that the channel varies between transmissions and results in channel aging. To model the time correlation between consecutive channel instantiations, the time-variant channel vector at the time instant $n$ after the most recent channel estimation can be given by

$$
\begin{aligned}
\mathbf{h}_{X R, k}(n) & =\rho \mathbf{h}_{X R, k}(n-1)+\mathbf{e}_{X R, k}(n) \\
= & \rho^{n-1} \mathbf{h}_{X R, k}(1)+\sum_{i=2}^{n} \rho^{n-i} \mathbf{e}_{X R, k}(i),
\end{aligned}
$$

where $\rho \in[0,1]$ is the time correlation parameter and $\mathbf{e}_{X R, k}(n)$ is the channel aging error at the time instant $n$ with zero mean and $\sigma_{e_{X R, k}}^{2}(n)$. For notational simplicity, we assume that all channel vectors experience the same time correlation parameter. In Eqn. (1), we consider that $\mathbf{g}_{X R, k}$ varies between transmissions and $\mathbf{A}_{k}$ is assumed to be fixed during all time instants at $T_{X, k}, X=A, B, k=1, \ldots, K$. In addition, the channel matrix at the time instant $n$ can be further defined as $\mathbf{H}_{X R}(n)=\left[\mathbf{h}_{X R, 1}(n), \ldots, \mathbf{h}_{X R, K}(n)\right] \in \mathbb{C}^{M \times K}$, $X=A, B$.

\section{Signal Transmission}

As per the Multiple Access Broadcast (MABC) protocol [5], the two-way relayed D2D data transmission process is divided into two stages with equal time slots. At the time instant $n$, all devices transmit signals to the relay simultaneously in the Multiple Access Channel (MAC) phase and the received signal at the relay is given by [6], [9], [11]

$$
\mathbf{y}_{r}(n)=\sum_{k=1}^{K} \sum_{X=A, B}\left(\sqrt{p_{X, k}} \mathbf{h}_{X R, k}(n) s_{X R, k}(n)\right)+\mathbf{n}_{R}(n),
$$

where $s_{X R, k}(n)$ is the Gaussian signal with zero mean and unit power transmitted by the $k$-th device $T_{X, k}$ at the time instant $n, p_{X, k}$ is the average transmit power of $\mathrm{T}_{X, k}, X=$ $A, B . \mathbf{n}_{R}(n)$ is the vector of additive white Gaussian noise (AWGN) with elements satisfying (i.i.d) $\mathcal{C N}(0,1)$. Assuming low-complexity linear processing at the relay, the transformed signal can be given by

$$
\mathbf{z}_{r}(n)=\mathbf{F}_{M A C}(n) \mathbf{y}_{r}(n) .
$$

Here, $\mathbf{F}_{M A C}(n) \in \mathbb{C}^{2 K \times M}$ is the linear receiver matrix.

In the Broadcast Channel (BC) phase, the relay first decodes the received information, then encodes and broadcasts it to device pairs [6]. The linear precoding matrix $\mathbf{F}_{B C}(n) \in \mathbb{C}^{M \times 2 K}$ in the $\mathrm{BC}$ phase is applied to obtain the transmit signal of the relay

$$
\mathbf{y}_{t}(n)=\rho_{D F}(n) \mathbf{F}_{B C}(n) \mathbf{s}(n)
$$

where $\mathbf{s}(n)=\left[\mathbf{s}_{A}^{T}(n), \mathbf{s}_{B}^{T}(n)\right]^{T}$ denotes the decoded signal at the time instant $n$ and $\rho_{D F}(n)$ is the normalization coefficient determined by the average relay power $\mathbb{E}\left\{\left\|\mathbf{y}_{t}(n)\right\|^{2}\right\}=p_{r}$. With the standard AWGN $n_{X, k}(n) \sim \mathcal{C N}(0,1)$, the received signal at the $k$-th device $T_{X, k}(k=1, \ldots, K, X=A, B)$ is given by

$$
z_{X, k}(n)=\mathbf{h}_{X R, k}^{T}(n) \mathbf{y}_{t}(n)+n_{X, k}(n) .
$$

\section{Linear Processing and Channel Estimation}

To achieve low complexity transmission, linear processing methods are applied at the relay. In this paper, we only take maximum ratio combining/maximum ratio transmission (MRC/MRT) processing into consideration. Other methods including zero forcing will be considered in the future studies. Therefore, the respective MRC/MRT processing matrices $\mathbf{F}_{M A C}(n) \in \mathbb{C}^{2 K \times M}$ and $\mathbf{F}_{B C}(n) \in \mathbb{C}^{M \times 2 K}$ at the time instant $n$ can be defined as

$$
\begin{aligned}
\mathbf{F}_{M A C}(n) & =\left[\hat{\mathbf{H}}_{A R}(n), \hat{\mathbf{H}}_{B R}(n)\right]^{H}, \\
\mathbf{F}_{B C}(n) & =\left[\hat{\mathbf{H}}_{B R}(n), \hat{\mathbf{H}}_{A R}(n)\right]^{*} .
\end{aligned}
$$




$$
\begin{aligned}
& C_{k}^{M A C}(n)=\sum_{X=A, B} p_{X, k}\left(\left|\hat{\mathbf{h}}_{X R, k}^{H}(n) \Delta \mathbf{h}_{X R, k}(n)\right|^{2}+\sum_{j=2}^{n}\left|\rho^{n-j} \hat{\mathbf{h}}_{X R, k}^{H}(n) \mathbf{e}_{X R, k}(j)\right|^{2}\right) \\
&+p_{A, k}\left(\left|\hat{\mathbf{h}}_{B R, k}^{H}(n) \Delta \mathbf{h}_{A R, k}(n)\right|^{2}+\sum_{j=2}^{n}\left|\rho^{n-j} \hat{\mathbf{h}}_{B R, k}^{H}(n) \mathbf{e}_{A R, k}(j)\right|^{2}\right) \\
&+p_{B, k}\left(\left|\hat{\mathbf{h}}_{A R, k}^{H}(n) \Delta \mathbf{h}_{B R, k}(n)\right|^{2}+\sum_{j=2}^{n}\left|\rho^{n-j} \hat{\mathbf{h}}_{A R, k}^{H}(n) \mathbf{e}_{B R, k}(j)\right|^{2}\right), \\
& D_{k}^{M A C}(n)=\sum_{i \neq k} \sum_{X=A, B}\left\{p_{A, i}\left[\left|\hat{\mathbf{h}}_{X R, k}^{H}(n) \hat{\mathbf{h}}_{A R, i}(n)\right|^{2}+\left|\hat{\mathbf{h}}_{X R, k}^{H}(n) \Delta \mathbf{h}_{A R, i}(n)\right|^{2}+\sum_{j=2}^{n}\left|\rho^{n-j} \hat{\mathbf{h}}_{X R, k}^{H}(n) \mathbf{e}_{A R, i}(j)\right|^{2}\right]\right\} \\
&+\sum_{i \neq k} \sum_{X=A, B}\left\{p_{B, i}\left[\left|\hat{\mathbf{h}}_{X R, k}^{H}(n) \hat{\mathbf{h}}_{B R, i}(n)\right|^{2}+\left|\hat{\mathbf{h}}_{X R, k}^{H}(n) \Delta \mathbf{h}_{B R, i}(n)\right|^{2}+\sum_{j=2}^{n}\left|\rho^{n-j} \hat{\mathbf{h}}_{X R, k}^{H}(n) \mathbf{e}_{B R, i}(j)\right|^{2}\right]\right\}, \\
& C_{k}^{B C}(n)=\sum_{i \neq k}\left[\left|\hat{\mathbf{h}}_{A R, k}^{T}(n) \hat{\mathbf{h}}_{A R, i}^{*}(n)\right|^{2}+\left|\Delta \mathbf{h}_{A R, k}^{T}(n) \hat{\mathbf{h}}_{A R, i}^{*}(n)\right|^{2}+\sum_{j=2}^{n}\left|\rho^{n-j} \mathbf{e}_{A R, k}^{T}(j) \hat{\mathbf{h}}_{A R, i}^{*}(n)\right|^{2}\right] \\
&+\sum_{i=1}^{K}\left[\left|\hat{\mathbf{h}}_{A R, k}^{T}(n) \hat{\mathbf{h}}_{B R, i}^{*}(n)\right|^{2}+\left|\Delta \mathbf{h}_{A R, k}^{T}(n) \hat{\mathbf{h}}_{B R, i}^{*}(n)\right|^{2}+\sum_{j=2}^{n}\left|\rho^{n-j} \mathbf{e}_{A R, k}^{T}(j) \hat{\mathbf{h}}_{B R, i}^{*}(n)\right|^{2}\right]
\end{aligned}
$$

Here, $\hat{\mathbf{H}}_{X R}(n)$ represents the CSI estimates at the time instant $n, X=A, B$. For notational simplicity, we define that $\mathbf{F}_{M A C}^{A R}(n) \in \mathbb{C}^{K \times M}, \mathbf{F}_{M A C}^{B R}(n) \in \mathbb{C}^{K \times M}$ are the first $K$ rows and the rest $K$ rows of $\mathbf{F}_{M A C}(n)$, respectively. $\mathbf{F}_{B C}^{R B}(n) \in \mathbb{C}^{M \times K}, \mathbf{F}_{B C}^{R A}(n) \in \mathbb{C}^{M \times K}$ represent the first $K$ columns of and the second $K$ columns of $\mathbf{F}_{B C}(n)$, respectively.

Assuming a minimum mean square error (MMSE) estimator, we can obtain the channel estimates for all time instants. In this paper, we assume that CSI is only estimated at the time instant 1 and explore the SE degradation in the following time instants; therefore, the channel estimates at the time instant 1 can be expressed as

$$
\begin{aligned}
\mathbf{h}_{X R, k}(1) & =\hat{\mathbf{h}}_{X R, k}(1)+\Delta \mathbf{h}_{X R, k}(1) \\
& =\mathbf{A}_{k}^{H} \hat{\mathbf{g}}_{X R, k}(1)+\mathbf{A}_{k}^{H} \mathbf{q} \mathbf{q}_{X, k}(1)
\end{aligned}
$$

$\hat{\mathbf{h}}_{X R, k}(1)$ and $\Delta \mathbf{h}_{X R, k}(1)$ are the estimated channel vector and the estimation error vector at the $k$-th device respectively. Furthermore, the respective estimated channel matrix and estimation error matrix at the time instant 1 can be given by $\hat{\mathbf{H}}_{X R}(1)$ and $\Delta \mathbf{H}_{X R}(1)$. The elements in $\hat{\mathbf{g}}_{X R, k}(1)$ and $\mathbf{q}_{X R, k}(1)$ are Gaussian random variables with zero mean, variance $\sigma_{X R, k}^{2}(1)$ and $\sigma_{\Delta h_{X R, k}}^{2}(1), X=A, B, k=1, \ldots, K$, respectively. Supported by Eqn. (11) and the effect of time correlation defined in Eqn. (4), the estimated channel vectors and estimation error vectors of the $k$-th device $T_{X R, k}(X=A, B$, $k=1, \ldots, K)$ in the following time instants can be further simplified as

$$
\begin{aligned}
& \hat{\mathbf{h}}_{X R, k}(n)=\rho^{n-1} \hat{\mathbf{h}}_{X R, k}(1)=\rho^{n-1} \mathbf{A}_{k}^{H} \hat{\mathbf{g}}_{X R, k}(1), \\
& \Delta \mathbf{h}_{X R, k}(n)=\rho^{n-1} \Delta \mathbf{h}_{X R, k}(1)=\rho^{n-1} \mathbf{A}_{k}^{H} \mathbf{q}_{X R, k}(1),
\end{aligned}
$$

while the relevant channel estimates at the time instant $n$ could be obtained via Eqn. (4), (12)-(13) and the detailed expression can be given by

$$
\begin{aligned}
\mathbf{h}_{X R, k}(n) & =\rho^{n-1} \mathbf{A}_{k}^{H} \mathbf{q}_{X R, k}(1)+\rho^{n-1} \mathbf{A}_{k}^{H} \hat{\mathbf{g}}_{X R, k}(1) \\
& +\sum_{i=2}^{n} \rho^{n-i} \mathbf{e}_{X R, k}(i) .
\end{aligned}
$$

\section{Analytical Spectral Efficiency Performance}

\section{A. Numerical expressions of Spectral Efficiency}

In this section, we focus on the SE performance of the proposed system. First, in the MAC phase, the transformed signals are obtained by applying the linear processing matrix $\mathbf{F}_{M A C}(n)$ to the received signals at the time instant $n$. Thus, the power of desired signals at $\mathrm{T}_{X, k},(k=1, \ldots, K$, $X=A, B)$ at the time instant $n$ can be given by

$$
\begin{aligned}
\mathbb{X}_{k}^{M A C}(n) & =p_{X, k}\left|\hat{\mathbf{h}}_{A R, k}^{H}(n) \hat{\mathbf{h}}_{X R, k}(n)\right|^{2} \\
& +p_{X, k}\left|\hat{\mathbf{h}}_{B R, k}^{H}(n) \hat{\mathbf{h}}_{X R, k}(n)\right|^{2}
\end{aligned}
$$

where $\mathbb{X}=A, B$. The respective power of estimation and channel aging error, inter-device interference of the postprocessing signals $\mathbf{z}_{r}(n)$ for the $k$-th device pair at the time instant $n$ can be expressed as Eqns. (16), (17) on the top of this page and the compound noise power is given by

$$
E_{k}^{M A C}(n)=\left|\hat{\mathbf{h}}_{A R, k}^{H}(n)\right|^{2}+\left|\hat{\mathbf{h}}_{B R, k}^{H}(n)\right|^{2} .
$$

In the following, we define that $\chi(1)=\frac{\tau_{c}-\tau_{p}}{2 \tau_{c}}$ and $\chi(n)=\frac{1}{2}$ $(n \neq 1)$ with $\tau_{c}$ and $\tau_{p}$ representing the coherence interval length and pilot sequence length respectively, since CSI is only estimated at the time instant 1 . Additionally, supported by Eqns. (15)-(18) and the standard lower capacity bound with the worst-case uncorrelated additive noise [9], the respective 


$$
\begin{aligned}
& \tilde{A}_{k}^{M A C}(n)=p_{A, k} \rho^{4(n-1)}\left[\sigma_{A R, k}^{4}\left(\sum_{\substack{a=1 \\
a=1 \\
b \neq a}}^{L}|\Theta(a, b)|^{2}+\sum_{a=1}^{L} \sum_{b=1}^{L} \Theta(a, a) \Theta(b, b)\right)+\sigma_{A R, k}^{2} \sigma_{B R, k}^{2} \sum_{a=1}^{L} \sum_{b=1}^{L}|\Theta(a, b)|^{2}\right], \\
& \tilde{B}_{k}^{M A C}(n)=p_{B, k} \rho^{4(n-1)}\left[\sigma_{B R, k}^{4}\left(\sum_{a=1}^{L} \sum_{\substack{b=1 \\
b \neq a}}^{L}|\Theta(a, b)|^{2}+\sum_{a=1}^{L} \sum_{b=1}^{L} \Theta(a, a) \Theta(b, b)\right)+\sigma_{A R, k}^{2} \sigma_{B R, k}^{2} \sum_{a=1}^{L} \sum_{b=1}^{L}|\Theta(a, b)|^{2}\right], \\
& \tilde{C}_{k}^{M A C}(n)=\eta(n) \cdot\left[\left(p_{A, k} \sigma_{\Delta h_{A R, k}}^{2}+p_{B, k} \sigma_{\Delta h_{B R, k}}^{2}\right)+\sum_{j=2}^{n} \rho^{2(1-j)}\left(p_{A, k} \sigma_{e_{A R, k}}^{2}(j)+p_{B, k} \sigma_{e_{B R, k}}^{2}(j)\right)\right] \sum_{a=1}^{L} \sum_{b=1}^{L}|\Theta(a, b)|^{2}, \\
& \tilde{D}_{k}^{M A C}(n)=\eta(n) \cdot \sum_{i \neq k}\left[\left(p_{A, i} \beta_{A R, i}+p_{B, i} \beta_{B R, i}\right)+\sum_{j=2}^{n} \rho^{2(1-j)}\left(p_{A, i} \sigma_{e_{A R, i}}^{2}(j)+p_{B, i} \sigma_{e_{B R, i}}^{2}(j)\right)\right] \sum_{a=1}^{L} \sum_{b=1}^{L}|\Theta(a, b)|^{2},
\end{aligned}
$$

SE of the $k$-th device pair and the SE of the device $T_{X, k}$ ( $X=A, B, k=1, \ldots, K)$ to the relay are defined as

$$
\begin{aligned}
& R_{1, k}(n)=\chi(n) \times \\
& \mathbb{E}\left\{\log _{2}\left(1+\frac{\sum_{\mathbb{X}=A, B} \mathbb{X}_{k}^{M A C}(n)}{C_{k}^{M A C}(n)+D_{k}^{M A C}(n)+E_{k}^{M A C}(n)}\right)\right\} . \\
& R_{X R, k}(n)=\chi(n) \times \\
& \mathbb{E}\left\{\log _{2}\left(1+\frac{\mathbb{X}_{k}^{M A C}(n)}{C_{k}^{M A C}(n)+D_{k}^{M A C}(n)+E_{k}^{M A C}(n)}\right)\right\} .
\end{aligned}
$$

In the $\mathrm{BC}$ phase, $\mathbf{F}_{B C}(n)$ is applied to generate the transmit signal of the relay and then the received signals are obtained at $T_{X, k}$ at the time instant $n$. In the following, we take the relevant terms at $T_{A, k}(k=1, \ldots, K)$ for details. We can obtain the power of desired signal, estimation and channel aging error respectively,

$$
\begin{gathered}
A_{k}^{B C}(n)=\left|\hat{\mathbf{h}}_{A R, k}^{T}(n) \hat{\mathbf{h}}_{A R, k}^{*}(n)\right|^{2}, \\
B_{k}^{B C}(n)=\left|\Delta \mathbf{h}_{A R, k}^{T}(n) \hat{\mathbf{h}}_{A R, k}^{*}(n)\right|^{2} \\
+\sum_{j=2}^{n}\left|\rho^{n-j} \mathbf{e}_{A R, k}^{T}(j) \hat{\mathbf{h}}_{A R, k}^{*}(n)\right|^{2} .
\end{gathered}
$$

Meanwhile, the inter-device interference power is shown on the top of this page by Eqn. (23) and the noise power can be given by

$$
D_{k}^{B C}(n)=\frac{1}{\rho_{D F}^{2}(n)}=1 /\left(\sqrt{\frac{p_{r}}{\mathbb{E}\left\{\left\|\mathbf{F}_{B C}(n)\right\|_{\mathrm{F}}^{2}\right\}}}\right)^{2} .
$$

Hence, the SE of the relay to the $k$-th device $T_{A, k}$ at the time instant $n$ is given by

$$
\begin{aligned}
& R_{R A, k}(n)=\chi(n) \times \\
& \mathbb{E}\left\{\log _{2}\left(1+\frac{A_{k}^{B C}(n)}{B_{k}^{B C}(n)+C_{k}^{B C}(n)+D_{k}^{B C}(n)}\right)\right\} .
\end{aligned}
$$

Similarly, $R_{R B, k}(n)$ can be obtained by replacing AR, BR with BR, AR in Eqns. (21)-(25). The sum SE of the $k$-th device pair in the BC phase is denoted as the sum of the end-to-end SE from $T_{A, k}$ to $T_{B, k}$ and the end-to-end SE from $T_{B, k}$ to $T_{A, k}$, which can be expressed as

$$
\begin{aligned}
R_{2, k}(n) & =\min \left(R_{A R, k}(n), R_{R B, k}(n)\right) \\
& +\min \left(R_{B R, k}(n), R_{R A, k}(n)\right) .
\end{aligned}
$$

Based to [6], [18], the sum SE of the multi-pair D2D twoway DF relaying system at the time instant $n$ can be obtained by

$$
R(n)=\sum_{k=1}^{K} \min \left(R_{1, k}(n), R_{2, k}(n)\right) .
$$

The average SE of the whole transmission process with channel aging can be further given by

$$
R_{\text {avr }}=\frac{1}{N} \sum_{n=1}^{N} R(n),
$$

where $N$ is the number of total time instants.

\section{B. Large-scale Approximations of Spectral Efficiency}

Note that $\hat{\mathbf{G}}_{X R}(1)=\left[\hat{\mathbf{g}}_{X R, 1}(1), \ldots, \hat{\mathbf{g}}_{X R, K}(1)\right] \in \mathbb{C}^{M \times D_{k}}$ based on Eqn. (11), $X=A, B$. When $M$ increases to a large number, the vectors in $\hat{\mathbf{G}}_{X R}(1)$ could be assumed to become asymptotically mutually orthogonal. Therefore, $\hat{\mathbf{G}}_{X R}^{H}(1) \hat{\mathbf{G}}_{X R}(1)$ approaches a diagonal matrix [19]. To simplify the simulations, we also assume that $\Theta=\mathbf{A A}^{\mathrm{H}}$ and $\mathbf{A}_{k}=\mathbf{A}_{j}, k, j=1, \ldots, K$. In this case, the approximations of corresponding SE terms in the MAC phase at the time instant $n$ defined in Sec. III-A can be given on the top of this page, while the approximation of the compound noise power is given by

$$
\tilde{E}_{k}^{M A C}(n)=\rho^{2(n-1)}\left(\sigma_{A R, k}^{2}+\sigma_{B R, k}^{2}\right) \cdot \sum_{a=1}^{L} \Theta(a, a) .
$$




$$
\begin{gathered}
\tilde{A}_{k}^{B C}(n)=\rho^{4(n-1)} \cdot \sigma_{A R, k}^{4}\left(\sum_{a=1}^{L} \sum_{b=1}^{L} \Theta^{*}(a, a) \Theta^{*}(b, b)+\sum_{a=1}^{L} \sum_{\substack{b=1 \\
b \neq a}}^{L}|\Theta(a, b)|^{2}\right) \\
\tilde{B}_{k}^{B C}(n)=\rho^{4(n-1)} \cdot \sigma_{A R, k}^{2}\left(\sigma_{\Delta h_{A R, k}}^{2}+\sum_{j=2}^{n} \rho^{2(1-j)} \sigma_{e_{A R, k}}^{2}(j)\right) \sum_{a=1}^{L} \sum_{b=1}^{L}|\Theta(a, b)|^{2} \\
\tilde{C}_{k}^{B C}(n)=\rho^{4(n-1)} \cdot\left\{\sigma_{A R, k}^{2}\left(\sum_{i \neq k} \beta_{A R, i}+\sum_{i=1}^{K} \beta_{B R, i}\right)+\sum_{j=2}^{n} \rho^{2(1-j)} \sigma_{e_{A R, k}}^{2}(j)\left(\sum_{i \neq k} \sigma_{A R, i}^{2}+\sum_{i=1}^{K} \sigma_{B R, i}^{2}\right)\right\} \sum_{a=1}^{L} \sum_{b=1}^{L}|\Theta(a, b)|^{2},
\end{gathered}
$$

Supported by Eqns. (18)-(19) and (28)-(32), we can obtain the SE approximations $\tilde{R}_{1, k}(n)$ and $\tilde{R}_{X R, k}(n), k=1, \ldots, K$, $X=A, B$. Meanwhile, in the $\mathrm{BC}$ phase, the approximated normalization coefficient $\tilde{\rho}_{D F}(n)$ can be expressed as

$$
\tilde{\rho}_{D F}(n)=\sqrt{\frac{p_{r}}{\rho^{2(n-1)} \sum_{j=1}^{K}\left(\sigma_{A R, j}^{2}+\sigma_{B R, j}^{2}\right) \sum_{m=1}^{L} \sum_{n=1}^{M}\left|\mathbf{A}_{m, n}\right|^{2}}}
$$

Moreover, the approximations of the $\mathrm{SE}$ terms at $T_{A R, k}$ in the BC phase at the time instant $n$ defined in Sec. IIIA can be given by on the top of next page with $\eta(n)=$ $\rho^{4(n-1)}\left(\sigma_{A R, k}^{2}+\sigma_{B R, k}^{2}\right)$, while the approximation of noise power can be expressed as

$\tilde{D}_{k}^{B C}(n)=\frac{\rho^{2(n-1)}}{p_{r}} \sum_{j=1}^{K}\left(\sigma_{A R, j}^{2}+\sigma_{B R, j}^{2}\right) \sum_{m=1}^{L} \sum_{n=1}^{M}\left|\mathbf{A}_{m, n}\right|^{2}$.

Therefore, $\tilde{R}_{R A, k}(n)$ can be obtained by Eqns. (25) and (34)-(38). Similarly, $\tilde{R}_{R B, k}(n)$ can be obtained by replacing $\mathrm{AR}, \mathrm{BR}$ with BR, AR in Eqns. (34)-(38). With the assistance of all approximation terms derived above, $\tilde{R}_{1, k}(n), \tilde{R}_{2, k}(n)$, $\tilde{R}(n)$ and $\tilde{R}_{a v r}$ can be obtained by Eqn. (19)-(20), (25)-(28).

\section{Performance Results}

In this section, we compare our analytical derivations with simulation results to verify the SE analysis of the proposed space-constrained D2D two-way relaying system with channel aging. The large-scale fading parameters are assumed to be $\beta_{A R, k}=\beta_{B R, k}=1$ and the device transmit power is considered as $p_{A, k}=p_{B, k}=p_{u}, k=1, \ldots, K$. For simplicity, we consider that $p_{p}=p_{u}$ and $p_{r}$ have fixed values in our simulations, the effect of the transmitted power values would be taken into consideration in the future work. Based on the LTE standard, the coherence interval length is $\tau_{c}=196$ (symbols) and the required pilot sequence length is $\tau_{p}=2 K$. The number of angular directions is $L=200$ and the angle spreads of the azimuth and elevation angles of departure are fixed to $\pi / 4$ and $\pi / 3$ in space-constrained massive MIMO respectively. For the imperfect channel estimation, the CSI is only estimated at the time instant 1 with $\sigma_{X R, k}^{2}(1)=\frac{10}{11}$, $\sigma_{\Delta h_{X R, k}}^{2}(1)=\frac{1}{11}, X=A, B, k=1, \ldots, K$. For channel aging, the variance of channel aging error is $\sigma_{e_{X R, k}}^{2}(n)=0.1$

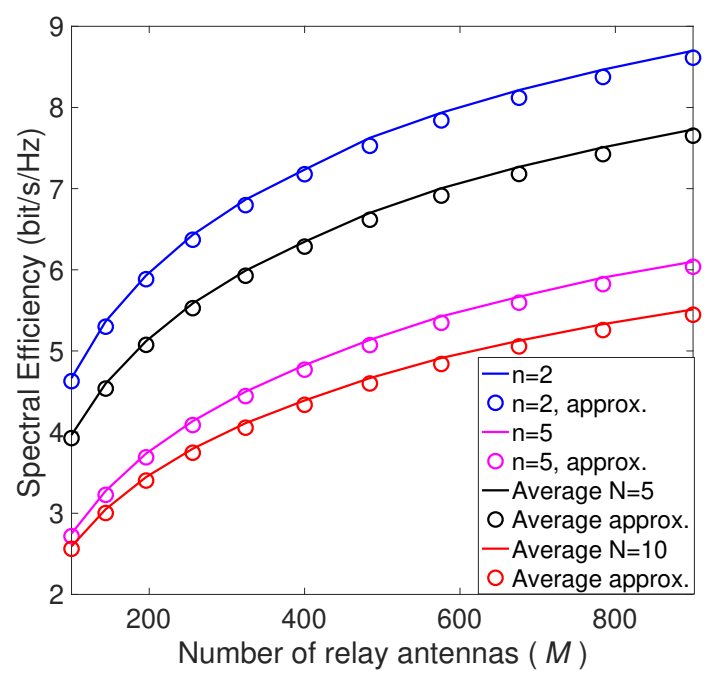

Fig. 2. SE v.s. number of relay antennas for $K=5, d=0.3 \lambda, p_{p}=p_{u}=0$ $\mathrm{dB}$ and $p_{r}=10 \mathrm{~dB}$.
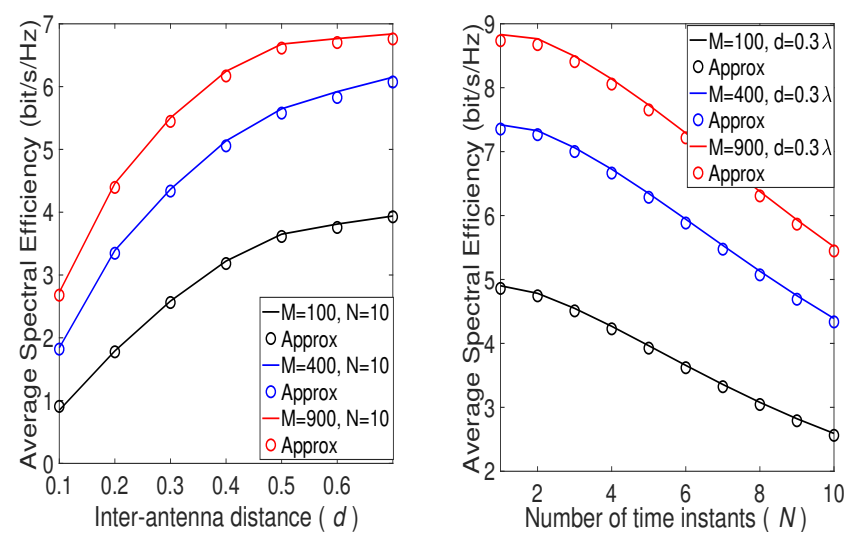

Fig. 3. Average SE v.s. a) inter-antenna distance and b) number of time instants for $K=5, p_{p}=p_{u}=0 \mathrm{~dB}$ and $p_{r}=10 \mathrm{~dB}$.

( $X=A, B, k=1, \ldots, K)$ in all time instants and the time correlation parameter is $\rho=0.8$ until otherwise stated.

Fig. 2 shows the instantaneous SE at the selected time instant $n$ after the most recent channel estimation and the average SE for all time instants vs the number of relay antennas respectively. The "Approx." (Approximations) are 


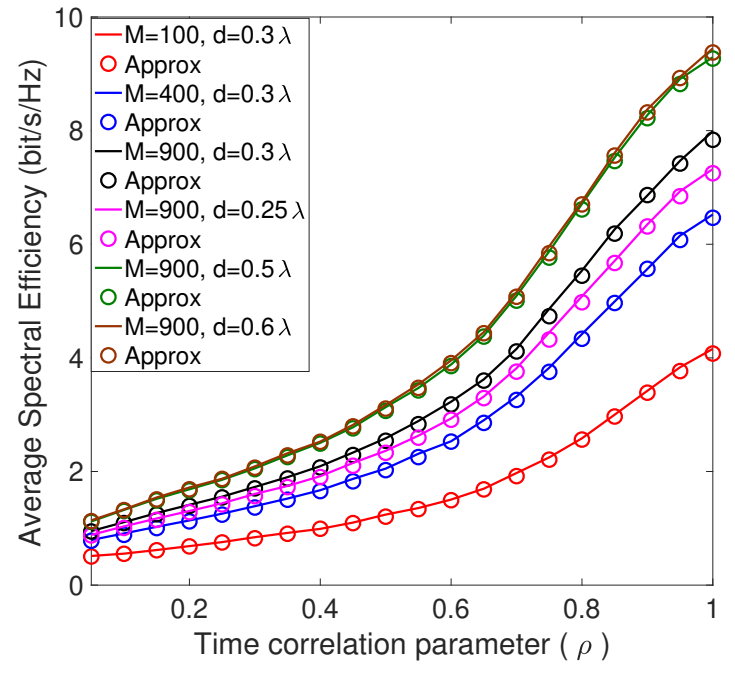

Fig. 4. Average SE v.s. time correlation parameter for $K=5, N=10$, $p_{p}=p_{u}=0 \mathrm{~dB}$ and $p_{r}=10 \mathrm{~dB}$.

obtained by Eqns. (19)-(20), (25)-(38), and numerical results are generated by Eqns. (15)-(28). It can be observed that the large-scale approximations can match the numerical results closely. We can also observe that both the average SE and the instantaneous SE for the selected time instant $n$ grow unboundedly with increasing $M$. Moreover, both larger $N$ and $n$ could reduce the average $\mathrm{SE}$ and the $\mathrm{SE}$ at the selected time instant due to the effect of channel aging respectively.

Fig. 3, the direct effect of spatial and time correlation is explored, where we show the average SE with increasing interantenna distance and the total number of time instants, with the CSI estimated at the time instant 1. Similar to Fig.2, larger $M$ can help to achieve a better SE performance. The average SE increases with inter-antenna distance and starts to saturate especially when $d \geq 0.5 \lambda$ with moderate spatial correlation. Meanwhile, the total number of time instants plays a negative role on the average SE. Nevertheless, it is important to note that significant performance degradation only appears for interantenna spacing $d<0.3 \lambda$, and for channel aging more than 4 to 5 time instants. This can be exploited for significant physical space savings and channel estimation relaxation.

In Fig. 4, the effect of the time correlation parameter on the average SE is illustrated. It is obviously shown that the average SE would increase with growing $\rho$ which represents increasingly retained channel accuracy and similar to Fig. 2, larger $M$ has positive effects on the average SE. Moreover, Fig. 4 can further clarify that increasing $d$ has positive effects, however when $d$ is large enough to achieve moderate even low spatial correlation, the average SE could tend to saturate. This can show that the joint spatial and time correlation could be tolerated in terms of the SE performance.

\section{Conclusion}

In this paper, we have studied the SE performance of a space-constrained multi-pair D2D two-way massive MIMO half-duplex DF relaying system with MRC/MRT processing, imperfect CSI and channel aging. The closed-form large-scale approximations of the SE are investigated with the impact of spatial correlation and channel aging. Supported by the approximations, our analysis shows that the moderate spatial and time correlation can be tolerated at the relay in the massive MIMO regime. In this case, both space savings and channel estimation relaxation can be exploited to maintain system performance while reducing costs for the D2D communications in the IoT environments.

\section{REFERENCES}

[1] J. Huang and H. Gharavi, "Performance analysis of relay-based twoway D2D communications with network coding," IEEE Transactions on Vehicular Technology, vol. 67, no. 7, pp. 6642-6646, 2018.

[2] L. Melki, S. Najeh, and H. Besbes, "System performance of two-way decode-and-forward relaying assisted D2D communication underlaying cellular networks," in 2016 International Symposium on Signal, Image, Video and Communications (ISIVC), 2016, pp. 270-275.

[3] J. Feng et al., "Sum rate of full-duplex two-way massive MIMO relay systems with channel aging," in 2016 IEEE ICCS, Dec 2016, pp. 1-6.

[4] H. Cui et al., "Multi-pair two-way amplify-and-forward relaying with very large number of relay antennas," IEEE Trans. Wireless Commun., vol. 13, no. 5, pp. 2636-2645, May 2014.

[5] S. J. Kim et al., "Achievable rate regions and performance comparison of half duplex bi-directional relaying protocols," IEEE Trans. Inf. Theory, vol. 57, no. 10, pp. 6405-6418, Oct 2011.

[6] C. Kong et al., "Multipair two-way half-duplex DF relaying with massive arrays and imperfect CSI," IEEE Trans. Wireless Commun., vol. 17, no. 5, pp. 3269-3283, May 2018.

[7] Z. Zhang et al., "Spectral and energy efficiency of multipair two-way full-duplex relay systems with massive MIMO," IEEE J. Sel. Areas Commun., vol. 34, no. 4, pp. 848-863, April 2016.

[8] H. Q. N. andothers, "Multipair full-duplex relaying with massive arrays and linear processing," IEEE J. Sel. Areas Commun., vol. 32, no. 9, pp. 1721-1737, Sept 2014.

[9] J. Qian et al., "Multi-pair two-way massive MIMO relaying with zero forcing: Energy efficiency and power scaling laws," IEEE Trans. Commun., pp. 1-1, 2019.

[10] L. Li et al., "Spectral- and energy-efficiency of multi-pair two-way massive MIMO relay systems experiencing channel aging," IEEE Access, vol. 7, pp. 46014-46032, 2019.

[11] J. He et al., "Multi-pair bidirectional relaying with full-duplex massive MIMO experiencing channel aging," in 2017 IEEE GLOBECOM, Dec 2017, pp. 1-6.

[12] S. Biswas et al., "Performance analysis of large multiuser MIMO systems with space-constrained 2-D antenna arrays," IEEE Trans. Wireless Commun., vol. 15, no. 5, pp. 3492-3505, May 2016.

[13] P. V. Amadori and C. Masouros, "Large scale antenna selection and precoding for interference exploitation," IEEE Trans. Commun., vol. 65, no. 10 , pp. 4529-4542, Oct 2017.

[14] H. Q. Ngo et al., "Massive MU-MIMO downlink TDD systems with linear precoding and downlink pilots," in 2013 51st Allerton, Oct 2013, pp. 293-298.

[15] N. Fatema et al., "Massive MIMO linear precoding: A survey," IEEE Systems Journal, pp. 1-12, 2018.

[16] J. Qian et al., "Partial CSI acquisition for size-constrained massive MIMO systems with user mobility," IEEE Trans. Veh. Tech., vol. 67, no. 9, pp. 9016-9020, Sep. 2018.

[17] A. Garcia-Rodriguez and C. Masouros, "Exploiting the increasing correlation of space constrained massive MIMO for CSI relaxation," IEEE Trans. Commun., vol. 64, no. 4, pp. 1572-1587, April 2016.

[18] B. Rankov and A. Wittneben, "Spectral efficient protocols for halfduplex fading relay channels," IEEE J. Sel. Areas Commun., vol. 25, no. 2, pp. 379-389, Feb 2007.

[19] C. Masouros and M. Matthaiou, "Space-constrained massive MIMO: Hitting the wall of favorable propagation," IEEE Commun. Lett., vol. 19, no. 5, pp. 771-774, May 2015. 